\title{
The European COST Action VascAgeNet Fostering Innovation - When Industry Comes to Science
}

\author{
Christopher C. Mayer ${ }^{1, *}{ }^{(\mathbb{D}}$, Rachel E. Climie ${ }^{2,3,4}$, Bernhard Hametner $^{1}$, Rosa-Maria Bruno ${ }^{2,5}$ \\ ${ }^{1}$ Center for Health \& Bioresources, Biomedical Systems, AIT Austrian Institute of Technology GmbH, Giefinggasse 4, Vienna 1210, Austria \\ ${ }^{2}$ INSERM U970 Paris-Centre de Recherche Cardiovasculaire (PARCC), Paris, France \\ ${ }^{3}$ Sports Cardiology and Diabetes and Population Health, Baker Heart and Diabetes Institute, Melbourne, Australia \\ ${ }^{4}$ Blood Pressure Research Group, Menzies Institute for Medical Research, College of Health and Medicine, \\ University of Tasmania, Hobart/Tasmania, Australia \\ ${ }^{5}$ Department of Pharmacology, Université Paris Descartes - Université de Paris, Paris, France
}

\section{ARTICLE INFO}

Article History

Received 29 November 2019

Accepted 28 April 2020

Keywords

COST action

VascAgeNet

vascular ageing

innovation

\begin{abstract}
VascAgeNet is a new European network which will join forces in order to refine, harmonize and promote the vascular ageing concept. It aims to bring innovations in cardiovascular research from bench to bedside and to establish assessment of vascular ageing in clinical practice. The interdisciplinary, inter-country and intersectional approach will enable all important players at the different stages of the innovation process to come together, as recommended in the triple or quadruple helix model of innovation, in order to guarantee best solutions. This is deemed necessary in order for economic and revolutionary solutions to be fit for wide-spread clinical use, especially in low-income and inclusiveness target countries. Thus, a clear case exists for a strong collaboration between science and industry, to foster innovations and breakthroughs in vascular ageing in order to improve clinical practice and to reduce the burden of cardiovascular diseases globally.
\end{abstract}

(c) 2020 Association for Research into Arterial Structure and Physiology. Publishing services by Atlantis Press International B.V. This is an open access article distributed under the CC BY-NC 4.0 license (http://creativecommons.org/licenses/by-nc/4.0/).

\section{INTRODUCTION TO VascAgeNet}

The concept of vascular ageing, defined as the development of a number of alterations in artery structure and function, induced by aging, accelerated by the integrated effect of genetic and environmental factors and associated with cardiovascular disease, is widely known and accepted [1,2]. Arterial stiffness is one of the clinical manifestations of vascular ageing. Over the last two decades, a mounting body of evidence has been gathered to demonstrate the potential of arterial stiffness as a risk predictor for cardiovascular morbidity and mortality [3,4], and as an appealing therapeutic target [5]. However, despite of the mounting evidence and recent advances in the assessment of vascular ageing and arterial stiffness, unmet needs remain which hinder wide-spread use in clinical practice and accentuation in current guidelines for hypertension management. These unmet needs include the complexity of use and heterogeneity of approaches (i.e., lack of easy to use, affordable equipment), and insufficient validation in clinical settings, as none of the available biomarkers fulfills all of the criteria to be considered a surrogate endpoint [6]. Furthermore, research in this domain is performed in Europe and worldwide, but mainly independently in specialized research labs. Thus, the aim of the COST Action VascAgeNet (CA 18216; https://www.cost.eu/actions/CA18216/) is to establish a

"Corresponding author. Email: christopher.mayer@ait.ac.at Peer review under responsibility of the Association for Research into Arterial Structure and Physiology network which will refine, harmonize and promote the use of vascular ageing measures, in order to improve clinical practice and to reduce the burden of cardiovascular diseases globally.

VascAgeNet will "refine" already existing as well as novel, easyto-use technologies for the diagnosis, prevention, treatment and monitoring of vascular ageing; "harmonize" knowledge and data to complete clinical validation of the most established surrogate endpoints, including head-to-head comparisons of different techniques and identify gaps and; "promote" a vascular ageing culture and propagate the use of technologies and preventative strategies. To achieve these ambitious goals, VascAgeNet will bring science and industry together to allow for a knowledge transfer for better technology in cardiovascular research and to translate research from bench to bedside.

The work plan is especially designed for a close collaboration between all disciplines and players. The content-related Working Groups (WGs) will focus on physiological and technical background to refine the understanding on mechanisms of vascular ageing and to reach consensus of the application of models for the assessment of vascular ageing (WG2); technological aspects leading to standardization and head-to-head comparison of existing techniques and to validation of these (WG3) and; harmonization of existing data and research studies to form the foundations for large, interventional studies (WG4). WG2-4 will be accompanied by two capacity-building working groups (WG1 and WG5). For details, see Climie et al. [7]. 


\section{THE INNOVATION PROCESS IN CARDIOVASCULAR RESEARCH}

Innovation is derived from the Latin word innovare, which means "to innovate, to reform, to change" and thus is defined as "the introduction of something new" or "a new idea, method, or device" [8]. The innovation process describes the translation process from new and/or existing knowledge and technology to marketable solutions [9], thus is reflective of the translation from science to industry. There are several ways of describing the different stages of the innovation process, with one of the simplest being: (1) idea creation, (2) proof-of-concept, (3) development, and (4) market (adapted from Dörner et al. [10]).

There is consensus that best innovations result from cooperation between, and strategically consulting of specialized and complementary entities at all stages of the innovation process [11]. For the interaction between academia, industry and governments, in the 1990s, the triple helix model of innovation was developed and theorized to be a key component of any national or multi-national innovation strategy [12]. Etzowitz and Leydesdorff stated that "the focus on interactions between institutions of fundamental research on the supply side and corporations has not only been reflected in technology policies, but also in technology studies" [12]. The triple helix model is widely applied in different domains (e.g., $[13,14]$ ) and can also be easily translated to innovations in the cardiovascular (i.e., medical) domain. An extension of the triple helix model by including civil society as a fourth entity led to the quadruple helix model with the aim to bridge the gaps between innovation and civil society $[15,16]$.

For innovation in cardiovascular research, the following players are of importance: (1) basic Research and Development (R\&D), (2) industry, (3) clinical science, and, in an extended view, (4) the patients. Importantly, patients are involved and represented either via the clinicians or in a fourth dimension to ensure their acceptance of the innovation. The different players can be easily mapped to the concept of the triple or quadruple helix as can be seen in Figure 1. Thus, best innovation happens in the overlap representing the interaction of all involved players. This is demonstrated in detail for the triple helix (see Figure 1A) in the following (adapted from Eriksson et al. [13]): (1) if industry and basic research and development are the only factors, the outcome may not be widely accepted by clinicians and patients; (2) if only industry and clinical science (representing clinicians and patients) are working together, the result may be old-fashioned; (3) if only basic research and development and clinical science in turn are included, the solution may be uneconomical. This suggests that it is important that all players interact at the different stages of the innovation process to create highly accepted, innovative and cost-effective solutions.

Similarly, activities for the different players in the different stages of the innovation process can be defined, whereas a close collaboration and interaction at all stages is of uttermost importance. Figure 2 demonstrates example activities which are needed for a biomarker to fulfill all of the criteria to be considered a surrogate endpoint $[6,17]$. These activities range from the idea creation stage from basic research results or newly developed models by basic $\mathrm{R} \& \mathrm{D}$, to ideas based on available products and customer feedback from industry, and the input about physiological background and unmet clinical needs from clinical science representing clinicians and patients. In the proof-of-concept phase, first prototypes and feasibility studies are available, triggered by R\&D, taken-up and streamlined with the existing portfolio by industry, and tested for the first time to create data and first feedback by selected key-opinion-leaders. In the development stage, ease of use and translation to industry are targeted by $\mathrm{R} \& \mathrm{D}$, and industry needs to think about certification according to medical device regulations and about the development of suitable business models. At this stage, clinical science plays an important role regarding validation and demonstrating the biomarkers additional value and clinical utility. Finally, placing the medical device on the market leads to out-licensing of technology by $\mathrm{R} \& \mathrm{D}$; marketing and cost-effective analysis by industry, and further clinical research to prove clinical outcome and to provide reference values. These activities are just examples and do not claim completeness.

\section{HOW VascAgeNet IS SET UP FOR FOSTERING THE INNOVATION PROCESS FOR TECHNOLOGY TRANSFER IN CARDIOVASCULAR RESEARCH}

One of the main objectives of VascAgeNet is to create innovation in the cardiovascular domain with special emphasis on easy to use,
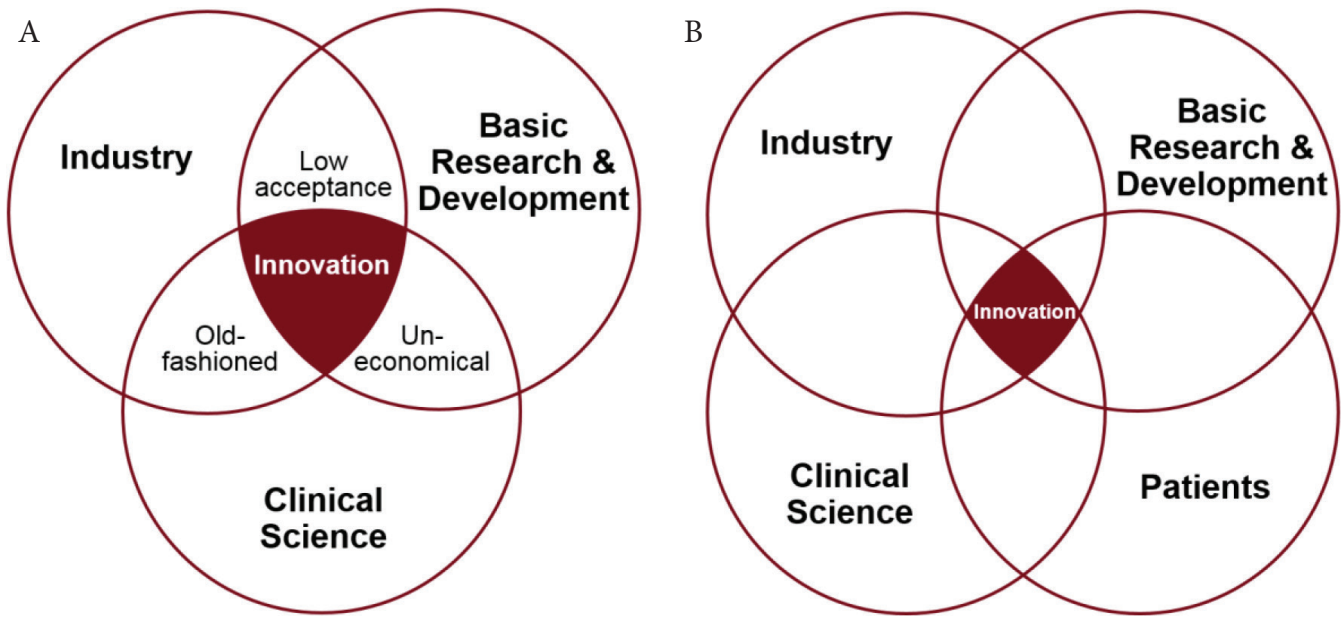

Figure 1 Triple helix (A) and quadruple helix (B) model of innovation applied to cardiovascular research (adapted from Eriksson et al. [13]). 


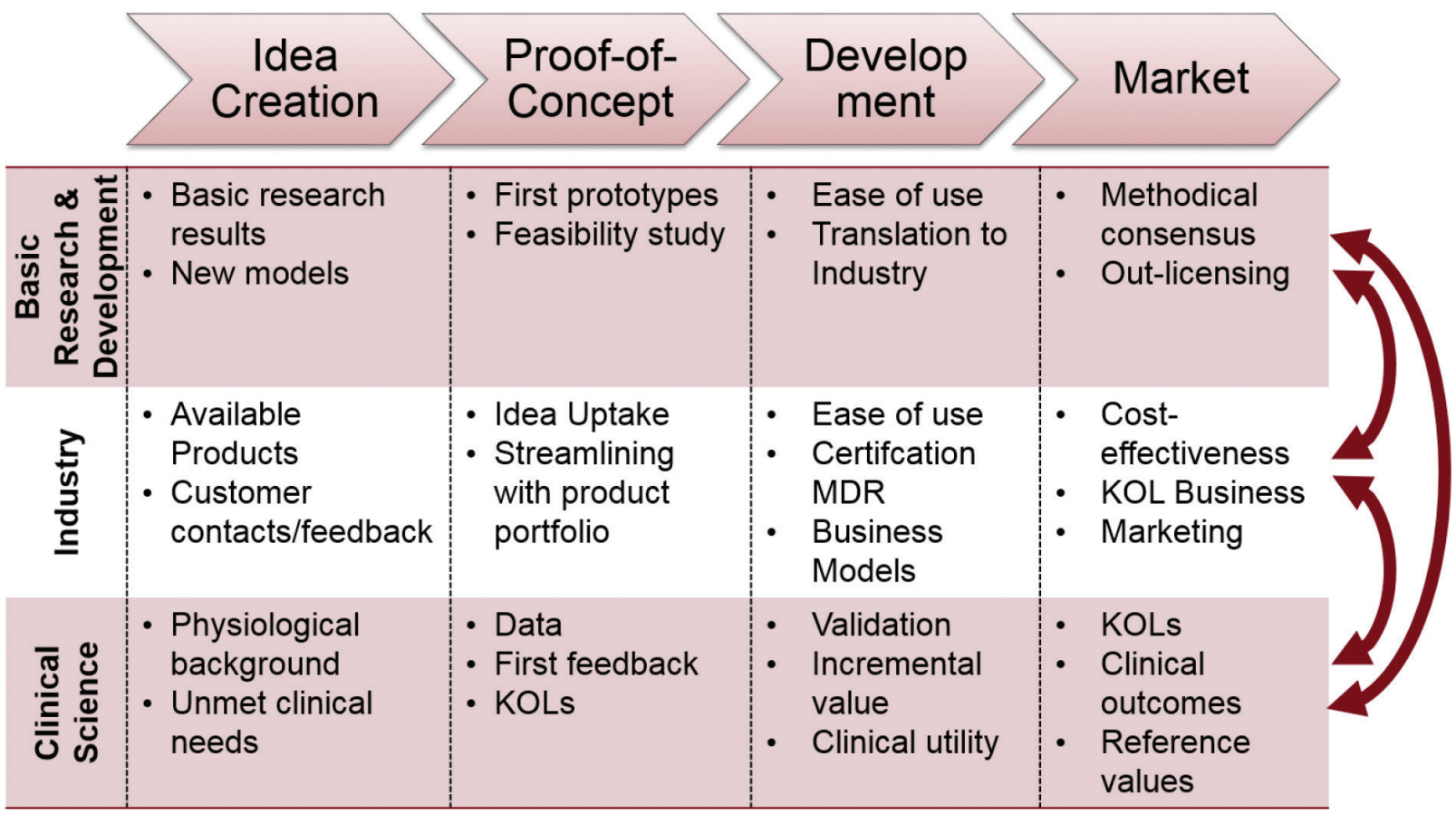

Figure 2 Examples of activities for the different players at the different stages of the innovation process in cardiovascular research. KOL, key-opinionleader; MDR, medical device regulation.

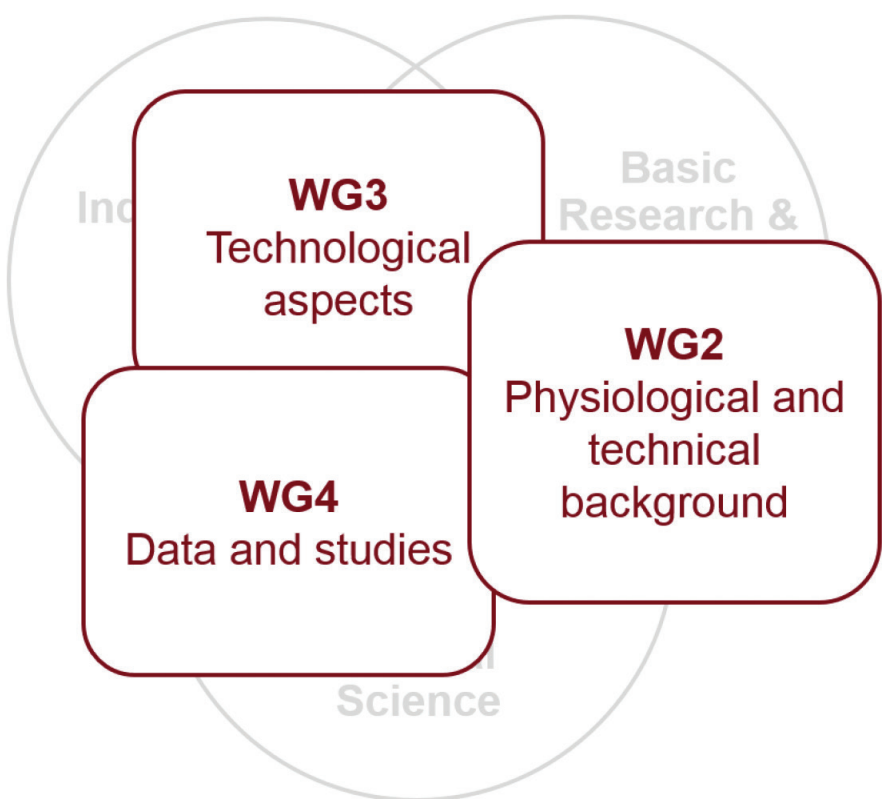

Figure 3 Mapping of the VascAgeNet Working Groups on the triple helix model of innovation.

affordable and validated equipment. This will be achieved by coordinating ongoing research in an interdisciplinary, inter-country and intersectoral network, which aims to connect and promote knowledge transfer between researchers, practitioners, patients and companies [7]. Figure 3 shows that one of the aims of VascAgeNet is to stimulate collaboration between the important players in cardiovascular research in the different working groups, which are mapped on the triple helix model of innovation displayed in Figure 1A. This approach is necessary in order for new innovations to move from bench to bedside and to establish vascular ageing assessment in routine clinical practice. Similarly, all activities from the different players from the different stages of the innovation process (Figure 2) can be mapped to the activities planned for the WGs of VascAgeNet (Figure 4). For example, output from WG2 in the idea creation phase will be to summarize current knowledge on mechanisms of vascular ageing, derived from basic research, and on genetics of vascular ageing combining the knowledge and skills of basic research and physiological background from clinical science. Another example of the output of WG2 in the first innovation stage is to reach consensus on the application, differences, interplay, limitations and potential complementarity of different mathematical models and techniques used for vascular ageing assessment advancing their potential clinical application. This again combines the expertise of R\&D and industry. Other activities in the innovation process are covered in WG3. One of the most important tasks of this WG will be to harmonize and standardize techniques for vascular ageing assessment, which is directly related to the proof-of-concept stage. Another task that WG3 will deal with is the creation of a framework to support the development and validation of innovative techniques, thus is directly related to the development stage in terms of certification and validation, and as well important for subsequent cost-effectiveness analyses in the last stage of the innovation process. Again, not only industry will be involved, but also R\&D and clinical science to ensure the collaboration as suggested in the triple helix model. As already mentioned, WG4 is mainly dealing with data and clinical studies which are the foundations for validation, showing incremental value and clinical utility, clinical outcomes and reference values, which are part of the development and marketing stages of the innovation process. This will again require interaction between the different players. The important and continuous collaboration between the players and working groups will be ensured by an accompanying WG 


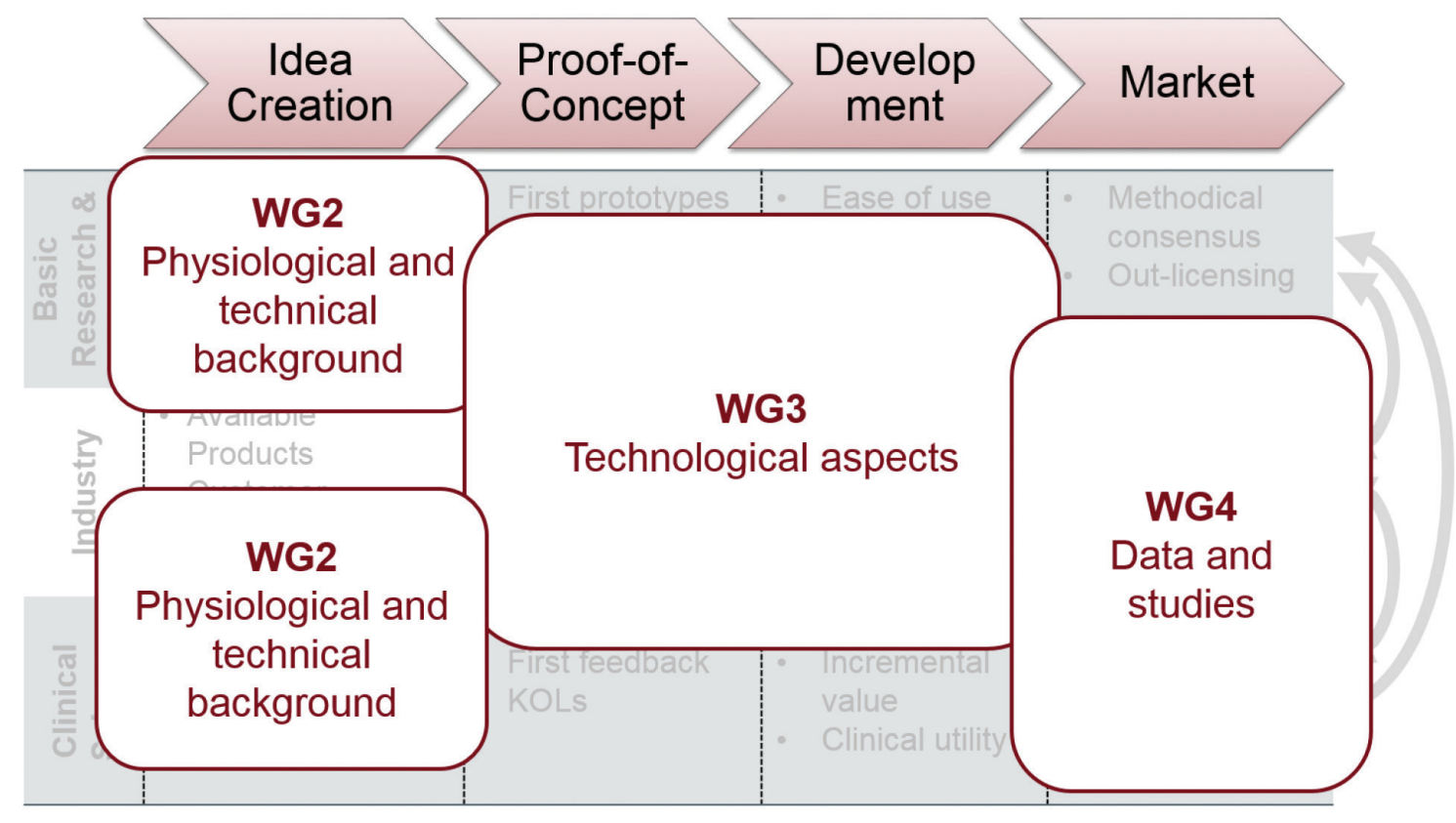

Figure 4 Mapping of the VascAgeNet Working Groups on the different activities in the innovation process.

focused on dynamic exchange (WG1; for details see Climie et al. [7]). Promotion of the concept of vascular ageing at the scientific level and also education of the broader community will be important to sustain the collaborations between science and technology. Thus, dissemination of results from VascAgeNet and the general concept of vascular ageing will focus on the scientific community and clinicians, stakeholders including (European) guideline committees and societies, health care systems and insurers, patients and the general public (WG5; [7]).

\section{CONCLUSION}

To foster innovation, collaboration between different players at the different stages of the innovation process is not only important, but necessary. Despite mounting evidence for the usefulness of arterial stiffness and vascular ageing biomarkers for risk predication, these are currently not measured in clinical practice, due to lack of easy to use and validated technology. This is where science (i.e., clinical science, basic research and development) and industry should work together to overcome these barriers and remaining unmet needs. The collaboration of the different players in the innovation process offers a unique opportunity to take important steps forward. VascAgeNet will provide a platform for this fruitful exchange in the next 4 years.

\section{CONFLICTS OF INTEREST}

The authors declare they have no conflicts of interest.

\section{AUTHORS' CONTRIBUTION}

All authors contributed in important intellectual content during manuscript drafting or revision.

\section{ACKNOWLEDGMENTS}

COST Action CA18216 is supported by COST (European Cooperation in Science and Technology, www.cost.eu). COST is a funding agency for research and innovation networks. Their actions help connect research initiatives across Europe and enable scientists to grow their ideas by sharing them with their peers. This boosts their research, career and innovation.

\section{REFERENCES}

[1] Nilsson PM, Boutouyrie P, Laurent S. Vascular aging: a tale of EVA and ADAM in cardiovascular risk assessment and prevention. Hypertension 2009;54:3-10.

[2] Laurent S, Boutouyrie P, Cunha PG, Lacolley P, Nilsson PM. Concept of extremes in vascular aging. Hypertension 2019;74:218-28.

[3] Vlachopoulos C, Aznaouridis K, Stefanadis C. Prediction of cardiovascular events and all-cause mortality with arterial stiffness: a systematic review and meta-analysis. J Am Coll Cardiol 2010;55:1318-27.

[4] Ben-Shlomo Y, Spears M, Boustred C, May M, Anderson SG, Benjamin EJ, et al. Aortic pulse wave velocity improves cardiovascular event prediction: an individual participant meta-analysis of prospective observational data from 17,635 subjects. J Am Coll Cardiol 2014;63:636-46.

[5] Wu CF, Liu PY, Wu TJ, Hung Y, Yang SP, Lin GM. Therapeutic modification of arterial stiffness: an update and comprehensive review. World J Cardiol 2015;7:742-53

[6] Vlachopoulos C, Xaplanteris P, Aboyans V, Brodmann M, Cífková R, Cosentino F, et al. The role of vascular biomarkers for primary and secondary prevention. A position paper from the European Society of Cardiology Working Group on peripheral circulation: endorsed by the Association for Research into Arterial 
Structure and Physiology (ARTERY) Society. Atherosclerosis 2015;241:507-32.

[7] Climie RE, Mayer CC, Bruno RM, Hametner B. Addressing the unmet needs of measuring vascular ageing in clinical practice European COoperation in Science and Technology Action VascAgeNet. Artery Res 2019. Forthcoming 2020.

[8] Merriam-Webster - Definition of innovation. Available from: https://www.merriam-webster.com/dictionary/innovation (last accessed November 28, 2019).

[9] LEAD Innovation Managament. Available from: https://www. lead-innovation.com/en/innovation-process (last accessed November 28, 2019).

[10] Dörner N, Gurtner S, Schefczyk M. Overcoming resistance to innovations: an approach for the use of communication tools within the innovation process. Int J Technol Market 2009;4: 199-216.

[11] Kimatu JN. Evolution of strategic interactions from the triple to quad helix innovation models for sustainable development in the era of globalization. J Innov Entrepr 2016;5:16.
[12] Etzkowitz H, Leydesdorff L. The triple helix-universityindustry-government relations: a laboratory for knowledge-based economic development. EASST Rev 1995;14:14-19.

[13] Eriksson M, Niitamo VP, Kulkki S, Hribernik KA. Living labs as a multi-contextual R\&D methodology. In: 2006 IEEE International Technology Management Conference. Milan, Italy: IEEE; 2006, pp. $1-8$.

[14] Rodrigues C, Melo AI. The triple helix model as inspiration for local development policies: an experience-based perspective. Int J Urban Region Res 2013;37:1675-87.

[15] Carayannis EG, Campbell DFJ. 'Mode 3' and 'Quadruple Helix': toward a 21st century fractal innovation ecosystem. Int J Technol Manage 2009;46:201-34.

[16] Cavallini S, Soldi R, Friedl J, Volpe M. Using the quadruple helix approach to accelerate the transfer of research and innovation results to regional growth (Consortium Progress Consulting S.r.l. \& Fondazione FORMIT). Brussels: European Union; 2016.

[17] Morrow DA, de Lemos JA. Benchmarks for the assessment of novel cardiovascular biomarkers. Circulation 2007;115:949-52. 